\title{
GENERALIZATIONS OF MINKOWSKI AND BECKENBACH-DRESHER INEQUALITIES AND FUNCTIONALS ON TIME SCALES
}

\author{
RABIA BIBI ${ }^{1, *}$, ANEES UR RAHMAN ${ }^{2}$ AND MUHAMMAD SHAHZAD ${ }^{2}$ \\ ${ }^{1}$ Department of Mathematics, Abbottabad University of Science and Technology, Havelian, Abbottabad, \\ Pakistan \\ ${ }^{2}$ Department of Mathematics, Hazara University, Mansehra, Pakistan \\ ${ }^{*}$ Corresponding author: emaorr@gmail.email
}

\begin{abstract}
We generalize integral forms of the Minkowski inequality and Beckenbach-Dresher inequality on time scales. Also, we investigate a converse of Minkowski's inequality and several functionals arising from the Minkowski inequality and the Beckenbach-Dresher inequality.
\end{abstract}

\section{Introduction ANd PRELIMINARIES}

A time scale $\mathbb{T}$ is an arbitrary nonempty closed subset of the real numbers. The theory of time scales was introduced by Stefan Hilger [7] in order to unify the theory of difference equations and the theory of differential equations. For an introduction to the theory of dynamic equations on time scales, we refer to $[3,8]$. Martin Bohner and Gusein Sh. Guseinov [4,5] defined the multiple Riemann and multiple Lebesgue integration on time scales and compared the Lebesgue $\Delta$-integral with the Riemann $\Delta$-integral.

Let $n \in \mathbb{N}$ be fixed. For each $i \in\{1, \ldots, n\}$, let $\mathbb{T}_{i}$ denote a time scale and

$$
\Lambda^{n}=\mathbb{T}_{1} \times \ldots \times \mathbb{T}_{n}=\left\{t=\left(t_{1}, \ldots, t_{n}\right): t_{i} \in \mathbb{T}_{i}, 1 \leq i \leq n\right\}
$$

Received 2019-08-18; accepted 2019-09-24; published 2020-03-02.

2010 Mathematics Subject Classification. Primary 26D15; Secondary 26A51, 34N05.

Key words and phrases. Minkowski inequality, Beckenbach-Dresher Inequality, time scales integrals.

(c)2020 Authors retain the copyrights of their papers, and all open access articles are distributed under the terms of the Creative Commons Attribution License. 
an $n$-dimensional time scale. Let $\mu_{\Delta}$ be the $\sigma$-additive Lebesgue $\Delta$-measure on $\Lambda^{n}$ and $\mathcal{F}$ be the family of $\Delta$-measurable subsets of $\Lambda^{n}$. Let $E \in \mathcal{F}$ and $\left(E, \mathcal{F}, \mu_{\Delta}\right)$ be a time scale measure space. Then for a $\Delta$-measurable function $f: E \rightarrow \mathbb{R}$, the corresponding $\Delta$-integral of $f$ over $E$ will be denoted according to $[5,(3.18)]$ by

$$
\int_{E} f\left(t_{1}, \ldots, t_{n}\right) \Delta_{1} t_{1} \ldots \Delta_{n} t_{n}, \quad \int_{E} f(t) \Delta t, \quad \int_{E} f \mathrm{~d} \mu_{\Delta}, \quad \text { or } \quad \int_{E} f(t) \mathrm{d} \mu_{\Delta}(t) .
$$

By [5, Section 3], all theorems of the general Lebesgue integration theory, including the Lebesgue dominated convergence theorem, hold also for Lebesgue $\Delta$-integrals on $\Lambda^{n}$. Here we state Fubini's theorem for integrals on time scales. It is used in the proofs of our main results.

Theorem 1.1 (Fubini's theorem). Let $\left(X, \mathcal{M}, \mu_{\Delta}\right)$ and $\left(Y, \mathcal{L}, \nu_{\Delta}\right)$ be two finite-dimensional time scale measure spaces. If $f: X \times Y \rightarrow \mathbb{R}$ is a $\Delta$-integrable function and if we define the functions

$$
\varphi(y)=\int_{X} f(x, y) \mathrm{d} \mu_{\Delta}(x) \quad \text { for a.e. } y \in Y
$$

and

$$
\psi(x)=\int_{Y} f(x, y) \mathrm{d} \nu_{\Delta}(y) \quad \text { for a.e. } \quad x \in X,
$$

then $\varphi$ is $\Delta$-integrable on $Y$ and $\psi$ is $\Delta$-integrable on $X$ and

$$
\int_{X} \mathrm{~d} \mu_{\Delta}(x) \int_{Y} f(x, y) \mathrm{d} \nu_{\Delta}(y)=\int_{Y} \mathrm{~d} \nu_{\Delta}(y) \int_{X} f(x, y) \mathrm{d} \mu_{\Delta}(x) .
$$

Hölder's inequality and Minkowski's inequality and their converses for multiple integration on time scales were investigated in [1]. These inequalities hold for both Riemann integrals and Lebesgue integrals on time scales. For completeness, let us recall these inequalities from [1].

Theorem 1.2 (Hölder's inequality [1, Theorem 6.2]). For $p \neq 1$, define $q=p /(p-1)$. Let $\left(E, \mathcal{F}, \mu_{\Delta}\right)$ be a time scale measure space. Assume $w, f, g$ are nonnegative functions such that $w f^{p}$, wg $g^{q}$, wfg are $\Delta$-integrable on $E$. If $p>1$, then

$$
\int_{E} w(t) f(t) g(t) \mathrm{d} \mu_{\Delta}(t) \leq\left(\int_{E} w(t) f^{p}(t) \mathrm{d} \mu_{\Delta}(t)\right)^{1 / p} \times\left(\int_{E} w(t) g^{q}(t) \mathrm{d} \mu_{\Delta}(t)\right)^{1 / q} .
$$

If $0<p<1$ and $\int_{E} w g^{q} \mathrm{~d} \mu_{\Delta}>0$, or if $p<0$ and $\int_{E} w f^{p} \mathrm{~d} \mu_{\Delta}>0$, then (1.2) is reversed.

Theorem 1.3 (Minkowski's inequality [1, Theorem 7.2]). Let $\left(E, \mathcal{F}, \mu_{\Delta}\right)$ be a time scale measure space. For $p \in \mathbb{R}$, assume $w, f, g$, are nonnegative functions such that $w f^{p}, w g^{p}, w(f+g)^{p}$ are $\Delta$-integrable on E. If $p \geq 1$, then

$$
\left(\int_{E} w(t)(f(t)+g(t))^{p} \mathrm{~d} \mu_{\Delta}(t)\right)^{\frac{1}{p}} \leq\left(\int_{E} w(t) f^{p}(t) \mathrm{d} \mu_{\Delta}(t)\right)^{1 / p}+\left(\int_{E} w(t) g^{p}(t) \mathrm{d} \mu_{\Delta}(t)\right)^{1 / p}
$$

If $0<p<1$ or $p<0$, then (1.3) is reversed provided each of the two terms on the right-hand side is positive. 
Theorem 1.4 (Converse of Hölder's inequality [1, Theorem 11.3]). For $p \neq 1$, define $q=p /(p-1)$. Let $\left(E, \mathcal{F}, \mu_{\Delta}\right)$ be a time scale measure space. Assume $w, f, g$ are nonnegative functions such that $w f^{p}, w g^{q}$, $w f g$ are $\Delta$-integrable on E. Suppose

$$
0<m \leq f(t) g^{-q / p}(t) \leq M \quad \text { for all } t \in E .
$$

If $p>1$, then

$$
\int_{E} w(t) f(t) g(t) \mathrm{d} \mu_{\Delta}(t) \geq K(p, m, M)\left(\int_{E} w(t) f^{p}(t) \mathrm{d} \mu_{\Delta}(t)\right)^{1 / p} \times\left(\int_{E} w(t) g^{q}(t) \mathrm{d} \mu_{\Delta}(t)\right)^{1 / q},
$$

where

$$
K(p, m, M)=|p|^{1 / p}|q|^{1 / q} \frac{(M-m)^{1 / p}\left|m M^{p}-M m^{p}\right|^{1 / q}}{\left|M^{p}-m^{p}\right|} .
$$

If $0<p<1$ or $p<0$, then (1.4) is reversed provided either $\int_{E} w g^{q} \mathrm{~d} \mu_{\Delta}>0$ or $\int_{E} w f^{p} \mathrm{~d} \mu_{\Delta}>0$.

In [2] Bibi et al., obtain integral forms of Minkowski's and Beckenbach-Dresher inequality on time scales. In this paper we generalize these inequalities and investigate functional obtained from our new inequalities.

\section{Minkowski Inequalities}

Let $U_{l}\left(x_{1}, x_{2}, \ldots, x_{l}\right), V_{m}\left(x_{1}, x_{2}, \ldots, x_{m}\right), G_{k}\left(x_{1}, x_{2}, \ldots, x_{k}\right)$, are real valued functions of $l, m$, and $k$ variables, respectively. Let $\left(X, \mathcal{M}, \mu_{\Delta}\right)$ and $\left(Y, \mathcal{L}, \nu_{\Delta}\right)$ be time scale measure spaces. Then, throughout in the following sections, we use the following notations:

$$
\begin{aligned}
& U_{l}=U_{l}(x)=U_{l}\left(u_{1}(x), u_{2}(x), \ldots, u_{l}(x)\right) \\
& V_{m}=V_{m}(y)=V_{m}\left(v_{1}(y), v_{2}(y), \ldots, v_{m}(y)\right) \\
& F_{k}=F_{k}(x, y)=F_{k}\left(f_{1}(x, y), f_{2}(x, y), \ldots, f_{k}(x, y)\right),
\end{aligned}
$$

where $\left\{u_{i}(x)\right\}_{i=1}^{l},\left\{v_{i}(y)\right\}_{i=1}^{m},\left\{f_{i}(x, y)\right\}_{i=1}^{k}$, are defined on $X, Y$, and $X \times Y$, respectively. In the sequel, we assume that all occurring integrals are finite.

Theorem 2.1 (Integral Minkowski inequality). If $p \geq 1$, then

$$
\left[\int_{X}\left(\int_{Y} F_{k}(x, y) V_{m}(y) \mathrm{d} \nu_{\Delta}(y)\right)^{p} U_{l}(x) \mathrm{d} \mu_{\Delta}(x)\right]^{\frac{1}{p}} \leq \int_{Y}\left(\int_{X} F_{k}^{p}(x, y) U_{l}(x) \mathrm{d} \mu_{\Delta}(x)\right)^{\frac{1}{p}} V_{m}(y) \mathrm{d} \nu_{\Delta}(y)
$$

holds provided all integrals in (2.2) exists. If $0<p<1$ and

$$
\int_{X}\left(\int_{Y} F_{k} V_{m} \mathrm{~d} \nu_{\Delta}\right)^{p} U_{l} \mathrm{~d} \mu_{\Delta}>0, \quad \int_{Y} F_{k} V_{m} \mathrm{~d} \nu_{\Delta}>0
$$

holds, then (2.2) is reversed. If $p<0$ and (2.3) and

$$
\int_{X} F_{k}^{p} U_{l} \mathrm{~d} \mu_{\Delta}>0
$$

hold, then (2.2) is reversed as well. 
Proof. Let $p \geq 1$. Put

$$
H(x)=\int_{Y} F_{k}(x, y) V_{m}(y) \mathrm{d} \nu_{\Delta}(y)
$$

Now, by using Fubini's theorem (Theorem 1.1) and Hölder's inequality (Theorem 1.2) on time scales, we have

$$
\begin{aligned}
& \int_{X} H^{p}(x) U_{l}(x) \mathrm{d} \mu_{\Delta}(x)=\int_{X} H(x) H^{p-1}(x) U_{l}(x) \mathrm{d} \mu_{\Delta}(x) \\
& =\int_{X}\left(\int_{Y} F_{k}(x, y) V_{m}(y) \mathrm{d} \nu_{\Delta}(y)\right) H^{p-1}(x) U_{l}(x) \mathrm{d} \mu_{\Delta}(x) \\
& =\int_{Y}\left(\int_{X} F_{k}(x, y) H^{p-1}(x) U_{l}(x) \mathrm{d} \mu_{\Delta}(x)\right) V_{m}(y) \mathrm{d} \nu_{\Delta}(y) \\
& \leq \int_{Y}\left(\int_{X} F_{k}^{p}(x, y) U_{l}(x) \mathrm{d} \mu_{\Delta}(x)\right)^{\frac{1}{p}}\left(\int_{X} H^{p}(x) U_{l}(x) \mathrm{d} \mu_{\Delta}(x)\right)^{\frac{p-1}{p}} V_{m}(y) \mathrm{d} \nu_{\Delta}(y) \\
& =\int_{Y}\left(\int_{X} F_{k}^{p}(x, y) U_{l}(x) \mathrm{d} \mu_{\Delta}(x)\right)^{\frac{1}{p}} V_{m}(y) \mathrm{d} \nu_{\Delta}(y)\left(\int_{X} H^{p}(x) U_{l}(x) \mathrm{d} \mu_{\Delta}(x)\right)^{\frac{p-1}{p}}
\end{aligned}
$$

and hence

$$
\left(\int_{X} H^{p}(x) U_{l}(x) \mathrm{d} \mu_{\Delta}(x)\right)^{\frac{1}{p}} \leq \int_{Y}\left(\int_{X} F_{k}^{p}(x, y) U_{l}(x) \mathrm{d} \mu_{\Delta}(x)\right)^{\frac{1}{p}} V_{m}(y) \mathrm{d} \nu_{\Delta}(y) .
$$

For $p<0$ and $0<p<1$, the corresponding results can be obtained similarly.

Theorem 2.2 (Converse of integral Minkowski inequality). Suppose

$$
0<m \leq \frac{F_{k}(x, y)}{\int_{Y} F_{k}(x, y) V_{m}(y) \mathrm{d} \nu_{\Delta}(y)} \leq M \quad \text { for all } \quad x \in X, y \in Y
$$

If $p \geq 1$, then

$$
\begin{aligned}
& {\left[\int_{X}\left(\int_{Y} F_{k}(x, y) V_{m}(y) \mathrm{d} \nu_{\Delta}(y)\right)^{p} U_{l}(x) \mathrm{d} \mu_{\Delta}(x)\right]^{\frac{1}{p}}} \\
& \qquad K(p, m, M) \int_{Y}\left(\int_{X} F_{k}^{p}(x, y) U_{l}(x) \mathrm{d} \mu_{\Delta}(x)\right)^{\frac{1}{p}} V_{m}(y) \mathrm{d} \nu_{\Delta}(y)
\end{aligned}
$$

provided all integrals in (2.5) exist, where $K(p, m, M)$ is defined by (1.5). If $0<p<1$ and (2.3) holds, then (2.5) is reversed. If $p<0$ and (2.3) and (2.4) hold, then (2.5) is reversed as well.

Proof. Let $p \geq 1$. Put

$$
H(x)=\int_{Y} F_{k}(x, y) V_{m}(y) \mathrm{d} \nu_{\Delta}(y)
$$


Then by using Fubini's theorem (Theorem 1.1) and the converse Hölder inequality (Theorem 1.4) on time scales, we get

$$
\begin{aligned}
\int_{X} H^{p}(x) U_{l}(x) \mathrm{d} \mu_{\Delta}(x) & =\int_{X}\left(\int_{Y} F_{k}(x, y) V_{m}(y) \mathrm{d} \nu_{\Delta}(y)\right) H^{p-1}(x) U_{l}(x) \mathrm{d} \mu_{\Delta}(x) \\
= & \int_{Y}\left(\int_{X} F_{k}(x, y) H^{p-1}(x) U_{l}(x) \mathrm{d} \mu_{\Delta}(x)\right) V_{m}(y) \mathrm{d} \nu_{\Delta}(y) \\
\geq & K(p, m, M) \int_{Y}\left(\int_{X} F_{k}^{p}(x, y) U_{l}(x) \mathrm{d} \mu_{\Delta}(x)\right)^{1 / p} \\
& \times\left(\int_{X} H^{p}(x) U_{l}(x) \mathrm{d} \mu_{\Delta}(x)\right)^{\frac{p-1}{p}} V_{m}(y) \mathrm{d} \nu_{\Delta}(y) .
\end{aligned}
$$

Dividing both sides by $\left(\int_{X} H^{p}(x) U_{l}(x) \mathrm{d} \mu_{\Delta}(x)\right)^{\frac{p-1}{p}}$, we obtain $(2.5)$. For $0<p<1$ and $p<0$, the corresponding results can be obtained similarly.

Now we define the $r$ th power mean $M^{[r]}\left(F_{k}, \mu_{\Delta}\right)$ of the function $F_{k}$ with respect to the measure $\mu_{\Delta}$ by

$$
M^{[r]}\left(F_{k}, \mu_{\Delta}\right)= \begin{cases}\left(\frac{\int_{X} F_{k}^{r}(x, y) U_{l}(x) \mathrm{d} \mu_{\Delta}(x)}{\int_{X} U_{l}(x) \mathrm{d} \mu_{\Delta}(x)}\right)^{\frac{1}{r}} & \text { if } \quad r \neq 0, \\ \exp \left(\frac{\int_{X} \log F_{k}(x, y) U_{l}(x) \mathrm{d} \mu_{\Delta}(x)}{\int_{X} U_{l}(x) \mathrm{d} \mu_{\Delta}(x)}\right) & \text { if } \quad r=0,\end{cases}
$$

where $\int_{X} U_{l} \mathrm{~d} \mu_{\Delta}>0$.

Corollary 2.1. Let $0<s \leq r$. Then

$$
M^{[r]}\left(M^{[s]}\left(F_{k}, \mathrm{~d} \nu_{\Delta}\right), \mathrm{d} \mu_{\Delta}\right) \geq K\left(\frac{r}{s}, m, M\right) M^{[s]}\left(M^{[r]}\left(F_{k}, \mathrm{~d} \mu_{\Delta}\right), \mathrm{d} \nu_{\Delta}\right)
$$

Proof. By putting $p=r / s$ and replacing $F_{k}$ by $F_{k}^{s}$ in (2.5), raising to the power of $\frac{1}{s}$ and dividing by

$$
\left(\int_{X} U_{l}(x) \mathrm{d} \mu_{\Delta}(x)\right)^{\frac{1}{r}}\left(\int_{Y} V_{m}(y) \mathrm{d} \nu_{\Delta}(y)\right)^{\frac{1}{s}}
$$

we get the above result.

\section{Minkowski Functionals}

In this section, we will consider some functionals which arise from the Minkowski inequality. Similar results (but not for time scales measure spaces) can be found in [9].

Let $F_{k}$ and $V_{m}$ be fixed functions satisfying the assumptions of Theorem 2.1. Let us consider the functional $\mathrm{M}_{1}$ defined by

$$
\mathrm{M}_{1}\left(U_{l}\right)=\left[\int_{Y}\left(\int_{X} F_{k}^{p}(x, y) U_{l}(x) \mathrm{d} \mu_{\Delta}(x)\right)^{\frac{1}{p}} V_{m}(y) \mathrm{d} \nu_{\Delta}(y)\right]^{p}-\int_{X}\left(\int_{Y} F_{k}(x, y) V_{m}(y) \mathrm{d} \nu_{\Delta}(y)\right)^{p} U_{l}(x) \mathrm{d} \mu_{\Delta}(x),
$$


where $U_{l}$ is a nonnegative function on $X$ such that all occurring integrals exist. Also, if we fix the functions $F_{k}$ and $U_{l}$, then we can consider the functional

$$
\begin{aligned}
\mathrm{M}_{2}\left(V_{m}\right)=\int_{Y}\left(\int_{X} F_{k}^{p}(x, y) U_{l}(x) \mathrm{d} \mu_{\Delta}(x)\right)^{\frac{1}{p}} V_{m}(y) \mathrm{d} \nu_{\Delta}(y) & \\
- & {\left[\int_{X}\left(\int_{Y} F_{k}(x, y) V_{m}(y) \mathrm{d} \nu_{\Delta}(y)\right)^{p} U_{l}(x) \mathrm{d} \mu_{\Delta}(x)\right]^{\frac{1}{p}}, }
\end{aligned}
$$

where $V_{m}$ is a nonnegative function on $Y$ such that all occurring integrals exist.

Remark 3.1. (i) It is obvious that $\mathrm{M}_{1}$ and $\mathrm{M}_{2}$ are positive homogeneous, i.e., $\mathrm{M}_{1}\left(a U_{l}\right)=a \mathrm{M}_{1}\left(U_{l}\right)$, and $\mathrm{M}_{2}\left(a V_{m}\right)=a \mathrm{M}_{2}\left(V_{m}\right)$, for any $a>0$.

(ii) If $p \geq 1$ or $p<0$, then $\mathrm{M}_{1}\left(U_{l}\right) \geq 0$, and if $0<p<1$, then $\mathrm{M}_{1}\left(U_{l}\right) \leq 0$.

(iii) If $p \geq 1$, then $\mathrm{M}_{2}\left(V_{m}\right) \geq 0$, and if $p<1$ and $p \neq 0$, then $\mathrm{M}_{2}\left(V_{m}\right) \leq 0$.

Theorem 3.1. (i) If $p \geq 1$ or $p<0$, then $\mathrm{M}_{1}$ is superadditive. If $0<p<1$, then $\mathrm{M}_{1}$ is subadditive.

(ii) If $p \geq 1$, then $\mathrm{M}_{2}$ is superadditive. If $p<1$ and $p \neq 0$, then $\mathrm{M}_{2}$ is subadditive.

(iii) Suppose $U_{l 1}$ and $U_{l 2}$ are nonnegative functions such that $U_{l 2} \geq U_{l 1}$. If $p \geq 1$ or $p<0$, then

$$
0 \leq \mathrm{M}_{1}\left(U_{l 1}\right) \leq \mathrm{M}_{1}\left(U_{l 2}\right)
$$

and if $0<p<1$, then (3.1) is reversed.

(iv) Suppose $V_{m 1}$ and $V_{m 2}$ are nonnegative functions such that $V_{m 2} \geq V_{m 1}$. If $p \geq 1$, then

$$
0 \leq \mathrm{M}_{2}\left(V_{m 1}\right) \leq \mathrm{M}_{2}\left(V_{m 2}\right)
$$

and if $p<1$ and $p \neq 0$, then (3.2) is reversed.

Proof. First we show (i). We have

$$
\begin{aligned}
\mathrm{M}_{1}\left(U_{l 1}+U_{l 2}\right)-\mathrm{M}_{1}\left(U_{l 1}\right)-\mathrm{M}_{1}\left(U_{l 2}\right) & {\left[\int_{Y}\left(\int_{X} f^{p}(x, y)\left(U_{l 1}+U_{l 2}\right)(x) \mathrm{d} \mu_{\Delta}(x)\right)^{\frac{1}{p}} V_{m}(y) \mathrm{d} \nu_{\Delta}(y)\right]^{p} } \\
= & \int_{X}\left(\int_{Y} F_{k}(x, y) V_{m}(y) \mathrm{d} \nu_{\Delta}(y)\right)^{p}\left(U_{l 1}+U_{l 2}\right)(x) \mathrm{d} \mu_{\Delta}(x) \\
- & {\left[\int_{Y}\left(\int_{X} F_{k}^{p}(x, y) U_{l 1}(x) \mathrm{d} \mu_{\Delta}(x)\right)^{\frac{1}{p}} V_{m}(y) \mathrm{d} \nu_{\Delta}(y)\right]^{p} } \\
+ & \int_{X}\left(\int_{Y} F_{k}(x, y) V_{m}(y) \mathrm{d} \nu_{\Delta}(y)\right)^{p} U_{l 1}(x) \mathrm{d} \mu_{\Delta}(x) \\
& -\left[\int_{Y}\left(\int_{X} F_{k}^{p}(x, y) U_{l 2}(x) \mathrm{d} \mu_{\Delta}(x)\right)^{\frac{1}{p}} V_{m}(y) \mathrm{d} \nu_{\Delta}(y)\right]^{p}
\end{aligned}
$$




$$
\begin{aligned}
& +\int_{X}\left(\int_{Y} F_{k}(x, y) V_{m}(y) \mathrm{d} \nu_{\Delta}(y)\right)^{p} U_{l 2}(x) \mathrm{d} \mu_{\Delta}(x) \\
= & {\left[\int_{Y}\left(\int_{X} F_{k}^{p}(x, y)\left(U_{l 1}+U_{l 2}\right)(x) \mathrm{d} \mu_{\Delta}(x)\right)^{\frac{1}{p}} V_{m}(y) \mathrm{d} \nu_{\Delta}(y)\right]^{p} } \\
- & {\left[\int_{Y}\left(\int_{X} F_{k}^{p}(x, y) U_{l 1}(x) \mathrm{d} \mu_{\Delta}(x)\right)^{\frac{1}{p}} V_{m}(y) \mathrm{d} \nu_{\Delta}(y)\right]^{p} } \\
- & {\left[\int_{Y}\left(\int_{X} F_{k}^{p}(x, y) U_{l 2}(x) \mathrm{d} \mu_{\Delta}(x)\right)^{\frac{1}{p}} V_{m}(y) \mathrm{d} \nu_{\Delta}(y)\right]^{p} . }
\end{aligned}
$$

Using the Minkowski inequality (1.3) for integrals (Theorem 1.3) with $p$ replaced by $1 / p$, we have

$$
\mathrm{M}_{1}\left(U_{l 1}+U_{l 2}\right)-\mathrm{M}_{1}\left(U_{l 1}\right)-\mathrm{M}_{1}\left(U_{l 2}\right) \begin{cases}\geq 0 & \text { if } \quad p \geq 1 \text { or } p<0 \\ \leq 0 & \text { if } \quad 0<p \leq 1\end{cases}
$$

So, $\mathbf{M}_{1}$ is superadditive for $p \geq 1$ or $p<0$, and it is subadditive for $0<p \leq 1$. The proof of (ii) is similar: After a simple calculation, we have

$$
\begin{aligned}
\mathrm{M}_{2}\left(V_{m 1}+V_{m 2}\right)-\mathrm{M}_{2}\left(V_{m 1}\right)-\mathrm{M}_{2}\left(V_{m 2}\right) \\
=\left[\int_{X}\left(\int_{Y} F_{k}(x, y) V_{m 1}(y) \mathrm{d} \nu_{\Delta}(y)\right)^{p} U_{l}(x) \mathrm{d} \mu_{\Delta}(x)\right]^{\frac{1}{p}} \\
+\left[\int_{X}\left(\int_{Y} F_{k}(x, y) V_{m 2}(y) \mathrm{d} \nu_{\Delta}(y)\right)^{p} U_{l}(x) \mathrm{d} \mu_{\Delta}(x)\right]^{\frac{1}{p}} \\
-\left[\int_{X}\left(\int_{Y} F_{k}(x, y)\left(V_{m 1}+V_{m 2}\right)(y) \mathrm{d} \nu_{\Delta}(y)\right)^{p} U_{l}(x) \mathrm{d} \mu_{\Delta}(x)\right]^{\frac{1}{p}} .
\end{aligned}
$$

Using the Minkowski inequality (2.2) for integrals (Theorem 2.1), we have that this is nonnegative for $p \geq 1$ and nonpositive for $p<1$ and $p \neq 0$. Now we show (iii). If $p \geq 1$ or $p<0$, then using superadditivity and positivity of $\mathrm{M}_{1}, U_{l 2} \geq U_{l 1}$ implies

$$
\mathrm{M}_{1}\left(U_{l 2}\right)=\mathrm{M}_{1}\left(U_{l 1}+\left(U_{l 2}-U_{l 1}\right)\right) \geq \mathrm{M}_{1}\left(U_{l 1}\right)+\mathrm{M}_{1}\left(U_{l 2}-U_{l 1}\right) \geq \mathrm{M}_{1}\left(U_{l 1}\right)
$$

and the proof of $(3.1)$ is established. If $0<p<1$, then using subadditivity and negativity of $\mathrm{M}_{1}, U_{l 2} \geq U_{l 1}$ implies

$$
\mathrm{M}_{1}\left(U_{l 2}\right) \leq \mathrm{M}_{1}\left(U_{l 1}\right)+\mathrm{M}_{1}\left(U_{l 2}-U_{l 1}\right) \leq \mathrm{M}_{1}\left(U_{l 1}\right)
$$

The proof of (iv) is similar.

Remark 3.2. Put $X, Y \subseteq \mathbb{N}$, then for fixed $F_{k}$ and $U_{l}$, the function $\mathrm{M}_{2}$ has the form

$$
\mathrm{M}_{2}\left(V_{m 1}\right)=\sum_{j \in Y} V_{m 1}(j)\left(\sum_{i \in X} U_{l}(i) F_{k}(i, j)^{p}\right)^{1 / p}-\left(\sum_{i \in X} U_{l}(i)\left(\sum_{j \in Y} V_{m 1}(j) F_{k}(i, j)\right)^{p}\right)^{1 / p}
$$


where $f(i, j)=F_{k}(i, j) \geq 0$. If $p \geq 1$, then the mapping $\mathrm{M}_{2}$ is superadditive, and $V_{m 2}(j) \geq V_{m 1}(j)$ for all $j \in Y$ implies

$$
\begin{aligned}
0 & \leq \sum_{j \in Y} V_{m 1}(j)\left(\sum_{i \in X} U_{l}(i) F_{k}(i, j)^{p}\right)^{1 / p}-\left(\sum_{i \in X} U_{l}(i)\left(\sum_{j \in Y} V_{m 1}(j) F_{k}(i, j)\right)^{p}\right)^{1 / p} \\
& \leq \sum_{j \in Y} V_{m 2}(j)\left(\sum_{i \in X} U_{l}(i) F_{k}(i, j)^{p}\right)^{1 / p}-\left(\sum_{i \in X} U_{l}(i)\left(\sum_{j \in Y} V_{m 2}(j) F_{k}(i, j)\right)^{p}\right)^{1 / p}
\end{aligned}
$$

provided all occurring sums are finite.

Corollary 3.1. (i) Suppose $U_{l 1}$ and $U_{l 2}$ are nonnegative functions such that $C U_{l 2} \geq U_{l 1} \geq c U_{l 2}$, where $c, C \geq 0$. If $p \geq 1$ or $p<0$, then

$$
c \mathrm{M}_{1}\left(U_{l 2}\right) \leq \mathrm{M}_{1}\left(U_{l 1}\right) \leq C \mathrm{M}_{1}\left(U_{l 2}\right)
$$

and if $0<p<1$, then the above inequality is reversed.

(ii) Suppose $V_{m 1}$ and $V_{m 2}$ are nonnegative functions such that $C V_{m 2} \geq V_{m 1} \geq c V_{m 2}$, where $c, C \geq 0$. If $p \geq 1$, then

$$
c \mathrm{M}_{2}\left(V_{m 2}\right) \leq \mathrm{M}_{2}\left(V_{m 1}\right) \leq C \mathrm{M}_{2}\left(V_{m 2}\right)
$$

and if $p<1$ and $p \neq 0$, then the above inequality is reversed.

Corollary 3.2. If $V_{m 1}$ and $V_{m 2}$ are nonnegative functions such that $V_{m 2} \geq V_{m 1}$, then

$$
\begin{aligned}
& M^{[0]}\left(\int_{Y} F_{k}(x, y) V_{m 1}(y)\right.\left.\mathrm{d} \nu_{\Delta}(y), \mu_{\Delta}\right)-\int_{Y} M^{[0]}\left(F_{k}, \mu_{\Delta}\right) V_{m 1}(y) \mathrm{d} \nu_{\Delta}(y) \\
& \leq M^{[0]}\left(\int_{Y} F_{k}(x, y) V_{m 2}(y) \mathrm{d} \nu_{\Delta}(y), \mu_{\Delta}\right)-\int_{Y} M^{[0]}\left(F_{k}, \mu_{\Delta}\right) V_{m 2}(y) \mathrm{d} \nu_{\Delta}(y),
\end{aligned}
$$

where $M^{[0]}\left(F_{k}, \mu_{\Delta}\right)$ is defined in $(2.6)$.

The next result gives another property of $M_{1}$, but a similar result can also be stated for $M_{2}$.

Theorem 3.2. Let $\varphi:[0, \infty) \rightarrow[0, \infty)$ be a concave function. Suppose $U_{l 1}$ and $U_{l 2}$ are nonnegative functions such that

$$
\varphi \circ U_{l 1}, \quad \varphi \circ U_{l 2}, \quad \varphi \circ\left(\alpha U_{l 1}+(1-\alpha) U_{l 2}\right)
$$

are $\Delta$-integrable for $\alpha \in[0,1]$. If $p \geq 1$, then

$$
\mathrm{M}_{1}\left(\varphi \circ\left(\alpha U_{l 1}+(1-\alpha) U_{l 2}\right)\right) \geq \alpha \mathrm{M}_{1}\left(\varphi \circ U_{l 1}\right)+(1-\alpha) \mathrm{M}_{1}\left(\varphi \circ U_{l 2}\right)
$$

and if $0<p<1$, then the above inequality is reversed. 
Proof. We show this only for $p \geq 1$ as the other case follows similarly. Since $\varphi$ is concave, we have

$$
\left.\varphi\left(\alpha U_{l 1}+(1-\alpha) U_{l 2}\right)\right) \geq \alpha \varphi\left(U_{l 1}\right)+(1-\alpha) \varphi\left(U_{l 2}\right)
$$

Now, from (3.1) and (3.3), we have

$$
\begin{aligned}
\mathrm{M}_{1}\left(\varphi \circ\left(\alpha U_{l 1}+(1-\alpha) U_{l 2}\right)\right) & \geq \mathrm{M}_{1}\left(\alpha\left(\varphi \circ U_{l 1}\right)+(1-\alpha)\left(\varphi \circ U_{l 2}\right)\right) \\
& \geq \mathrm{M}_{1}\left(\alpha\left(\varphi \circ U_{l 1}\right)\right)+\mathrm{M}_{1}\left((1-\alpha)\left(\varphi \circ U_{l 2}\right)\right) \\
& \geq \alpha \mathrm{M}_{1}\left(\varphi \circ U_{l 1}\right)+(1-\alpha) \mathrm{M}_{1}\left(\varphi \circ U_{l 2}\right),
\end{aligned}
$$

and the proof is established.

Let $F_{k}, U_{l}$ and $V_{m}$ be fixed functions satisfying the assumptions of Theorem 2.1. Let us define functionals $\mathrm{M}_{3}$ and $\mathrm{M}_{4}$ by

$$
\mathrm{M}_{3}(A)=\left[\int_{Y}\left(\int_{A} F_{k}^{p}(x, y) U_{l}(x) \mathrm{d} \mu_{\Delta}(x)\right)^{\frac{1}{p}} V_{m}(y) \mathrm{d} \nu_{\Delta}(y)\right]^{p}-\int_{A}\left(\int_{Y} F_{k}(x, y) V_{m}(y) \mathrm{d} \nu_{\Delta}(y)\right)^{p} U_{l}(x) \mathrm{d} \mu_{\Delta}(x)
$$

and

$$
\mathrm{M}_{4}(B)=\int_{B}\left(\int_{X} F_{k}^{p}(x, y) U_{l}(x) \mathrm{d} \mu_{\Delta}(x)\right)^{\frac{1}{p}} V_{m}(y) \mathrm{d} \nu_{\Delta}(y)-\left[\int_{X}\left(\int_{B} F_{k}(x, y) V_{m}(y) \mathrm{d} \nu_{\Delta}(y)\right)^{p} U_{l}(x) \mathrm{d} \mu_{\Delta}(x)\right]^{\frac{1}{p}},
$$

where $A \subseteq X$ and $B \subseteq Y$.

The following theorem establishes superadditivity and monotonicity of the mappings $M_{3}$ and $M_{4}$.

Theorem 3.3. $\quad$ (i) Suppose $A_{1}, A_{2} \subseteq X$ and $A_{1} \cap A_{2}=\emptyset$. If $p \geq 1$ or $p<0$, then

$$
\mathrm{M}_{3}\left(A_{1} \cup A_{2}\right) \geq \mathrm{M}_{3}\left(A_{1}\right)+\mathrm{M}_{3}\left(A_{2}\right),
$$

and if $0<p<1$, then the above inequality is reversed.

(ii) Suppose $A_{1}, A_{2} \subseteq X$ and $A_{1} \subseteq A_{2}$. If $p \geq 1$ or $p<0$, then

$$
\mathrm{M}_{3}\left(A_{1}\right) \leq \mathrm{M}_{3}\left(A_{2}\right)
$$

and if $0<p<1$, then the above inequality is reversed.

(iii) Suppose $B_{1}, B_{2} \subseteq Y$ and $B_{1} \cap B_{2}=\emptyset$. If $p \geq 1$, then

$$
\mathrm{M}_{4}\left(B_{1} \cup B_{2}\right) \geq \mathrm{M}_{4}\left(B_{1}\right)+\mathrm{M}_{4}\left(B_{2}\right),
$$

and if $p<1$ and $p \neq 0$, then the above inequality is reversed.

(iv) Suppose $B_{1}, B_{2} \subseteq Y$ and $B_{1} \subseteq B_{2}$. If $p \geq 1$, then

$$
\mathrm{M}_{4}\left(B_{1}\right) \leq \mathrm{M}_{4}\left(B_{2}\right)
$$

and if $p<1$ and $p \neq 0$, then the above inequality is reversed. 
The proof of Theorem 3.3 is omitted as it is similar to the proof of Theorem 3.1.

Remark 3.3. For $p \geq 1$, if $S_{m}$ is a subset of $Y$ with $m$ elements and if $S_{m} \supseteq S_{m-1} \supseteq \ldots \supseteq S_{2}$, then we have

$$
\mathrm{M}_{4}\left(S_{m}\right) \geq \mathrm{M}_{4}\left(S_{m-1}\right) \geq \ldots \geq \mathrm{M}_{4}\left(S_{2}\right) \geq 0
$$

and $\mathrm{M}_{4}\left(S_{m}\right) \geq \max \left\{\mathrm{M}_{4}\left(S_{2}\right): S_{2}\right.$ is any subset of $S_{m}$ with 2 elements $\}$.

\section{Beckenbach-Dresher Inequalities}

Let $U_{l}, V_{m}, F_{k}$ be defined as in (4.1). Let $\mathcal{F}_{n}\left(x_{1}, x_{2}, \ldots, x_{n}\right), \mathcal{G}_{t}\left(x_{1}, x_{2}, \ldots, x_{t}\right)$ are real valued functions of $n$, and $t$ variables, respectively. Let $\left(X, \mathcal{M}, \mu_{\Delta}\right),\left(X, \mathcal{M}, \lambda_{\Delta}\right)$ and $\left(Y, \mathcal{L}, \nu_{\Delta}\right)$ be time scale measure spaces. Then, throughout in the following sections, we use the following notations:

$$
\begin{aligned}
& \mathcal{W}_{n}=\mathcal{W}_{n}(x)=\mathcal{W}_{n}\left(w_{1}(x), w_{2}(x), \ldots, w_{n}(x)\right), \\
& \mathcal{G}_{t}=\mathcal{G}_{t}(x, y)=\mathcal{G}_{t}\left(g_{1}(x, y), g_{2}(x, y), \ldots, g_{t}(x, y)\right),
\end{aligned}
$$

where $U_{l}$ and $\mathcal{W}_{n}$ are nonnegative functions on $X, V_{m}$ is a nonnegative function on $Y, F_{k}$ is a nonnegative function on $X \times Y$ with respect to the measure $\left(\mu_{\Delta} \times \nu_{\Delta}\right)$, and $\mathcal{G}_{t}$ is a nonnegative function on $X \times Y$ with respect to the measure $\left(\lambda_{\Delta} \times \nu_{\Delta}\right)$. In the sequel, we assume that all occurring integrals are finite.

Theorem 4.1. If

$$
s \geq 1, \quad q \leq 1 \leq p, \quad \text { and } \quad q \neq 0
$$

or

$$
s<0, \quad p \leq 1 \leq q, \quad \text { and } \quad p \neq 0
$$

then

$$
\begin{aligned}
\frac{\left[\int_{X}\left(\int_{Y} F_{k}(x, y) V_{m}(y) \mathrm{d} \nu_{\Delta}(y)\right)^{p} U_{l}(x) \mathrm{d} \mu_{\Delta}(x)\right]^{\frac{s}{p}}}{\left[\int_{X}\left(\int_{Y} \mathcal{G}_{t}(x, y) V_{m}(y) \mathrm{d} \nu_{\Delta}(y)\right)^{q} \mathcal{W}_{n}(x) \mathrm{d} \lambda_{\Delta}(x)\right]^{\frac{s-1}{q}}} & \\
& \leq \int_{Y} \frac{\left(\int_{X} F_{k}^{p}(x, y) U_{l}(x) \mathrm{d} \mu_{\Delta}(x)\right)^{\frac{s}{p}}}{\left(\int_{X} \mathcal{G}_{t}^{q}(x, y) \mathcal{W}_{n}(x) \mathrm{d} \lambda_{\Delta}(x)\right)^{\frac{s-1}{q}}} V_{m}(y) \mathrm{d} \nu_{\Delta}(y)
\end{aligned}
$$

provided all occurring integrals in (4.4) exist. If

$$
0<s \leq 1, \quad p \geq 1, \quad q \leq 1, \quad \text { and } \quad q \neq 0
$$

then (4.4) is reversed. 
Proof. Assume (4.2) or (4.3). By using the integral Minkowski inequality (2.2) and Hölder's inequality (1.2), we have

$$
\begin{aligned}
& \frac{\left[\int_{X}\left(\int_{Y} F_{k}(x, y) V_{m}(y) \mathrm{d} \nu_{\Delta}(y)\right)^{p} U_{l}(x) \mathrm{d} \mu_{\Delta}(x)\right]^{\frac{s}{p}}}{\left[\int_{X}\left(\int_{Y} \mathcal{G}_{t}(x, y) V_{m}(y) \mathrm{d} \nu_{\Delta}(y)\right)^{q} \mathcal{W}_{n}(x) \mathrm{d} \lambda_{\Delta}(x)\right]^{\frac{s-1}{q}}} \\
& \leq \frac{\left[\int_{Y}\left(\int_{X} F_{k}^{p}(x, y) U_{l}(x) \mathrm{d} \mu_{\Delta}(x)\right)^{\frac{1}{p}} V_{m}(y) \mathrm{d} \nu_{\Delta}(y)\right]^{s}}{\left[\int_{Y}\left(\int_{X} \mathcal{G}_{t}^{q}(x, y) \mathcal{W}_{n}(x) \mathrm{d} \lambda_{\Delta}(x)\right)^{\frac{1}{q}} V_{m}(y) \mathrm{d} \nu_{\Delta}(y)\right]^{s-1}} \\
& =\left[\int_{Y}\left(\left(\int_{X} F_{k}^{p}(x, y) U_{l}(x) \mathrm{d} \mu_{\Delta}(x)\right)^{\frac{s}{p}}\right)^{\frac{1}{s}} V_{m}(y) \mathrm{d} \nu_{\Delta}(y)\right]^{s} \\
& \quad \times\left[\int_{Y}\left(\left(\int_{X} \mathcal{G}_{t}^{q}(x, y) \mathcal{W}_{n}(x) \mathrm{d} \lambda_{\Delta}(x)\right)^{\frac{1-s}{q}}\right)^{\frac{1}{1-s}} V_{m}(y) \mathrm{d} \nu_{\Delta}(y)\right]^{1-s} \\
& \leq \int_{Y}\left(\int_{X} F_{k}^{p}(x, y) U_{l}(x) \mathrm{d} \mu_{\Delta}(x)\right)^{\frac{s}{p}}\left(\int_{X} \mathcal{G}_{t}^{q}(x, y) \mathcal{W}_{n}(x) \mathrm{d} \lambda_{\Delta}(x)\right)^{\frac{1-s}{q}} V_{m}(y) \mathrm{d} \nu_{\Delta}(y) .
\end{aligned}
$$

If (4.5) holds, then the reversed inequality in (4.4) can be proved in a similar way.

\section{Beckenbach-Dresher Functionals}

Let $F_{k}, \mathcal{G}_{t}, U_{l}, \mathcal{W}_{n}$ be fixed functions satisfying the assumptions of Theorem 4.1. We define the Beckenbach-Dresher functional $\mathrm{BD}\left(V_{m}\right)$ by

$$
\begin{aligned}
\operatorname{BD}\left(V_{m}\right)=\int_{Y} \frac{\left(\int_{X} F_{k}^{p}(x, y) U_{l}(x) \mathrm{d} \mu_{\Delta}(x)\right)^{\frac{s}{p}}}{\left(\int_{X} \mathcal{G}_{t}^{q}(x, y) \mathcal{W}_{n}(x) \mathrm{d} \lambda_{\Delta}(x)\right)^{\frac{s-1}{q}}} V_{m}(y) \mathrm{d} \nu_{\Delta}(y) \\
-\frac{\left[\int_{X}\left(\int_{Y} F_{k}(x, y) V_{m}(y) \mathrm{d} \nu_{\Delta}(y)\right)^{p} U_{l}(x) \mathrm{d} \mu_{\Delta}(x)\right]^{\frac{s}{p}}}{\left[\int_{X}\left(\int_{Y} \mathcal{G}_{t}(x, y) V_{m}(y) \mathrm{d} \nu_{\Delta}(y)\right)^{q} \mathcal{W}_{n}(x) \mathrm{d} \lambda_{\Delta}(x)\right]^{\frac{s-1}{q}}},
\end{aligned}
$$

where we suppose that all occurring integrals exist.

Theorem 5.1. If (4.2) or (4.3) holds, then

$$
\mathrm{BD}\left(V_{m 1}+V_{m 2}\right) \geq \mathrm{BD}\left(V_{m 1}\right)+\mathrm{BD}\left(V_{m 2}\right) .
$$

If $V_{m 2} \geq V_{m 1}$, then

$$
\mathrm{BD}\left(V_{m 1}\right) \leq \mathrm{BD}\left(V_{m 2}\right)
$$

If $C, c \geq 0$ and $C V_{m 2} \geq V_{m 1} \geq c V_{m 2}$, then

$$
C \mathrm{BD}\left(V_{m 2}\right) \geq \mathrm{BD}\left(V_{m 1}\right) \geq c \mathrm{BD}\left(V_{m 1}\right) .
$$

If (4.5) holds, then (5.1), (5.2) and (5.3) are reversed. 
Proof. Assume (4.2) or (4.3). Then we have

$$
\begin{aligned}
\mathrm{BD}\left(V_{m 1}+V_{m 2}\right)-\mathrm{BD}\left(V_{m 1}\right)-\mathrm{BD}\left(V_{m 2}\right) & \\
= & \frac{\left[\int_{X}\left(\int_{Y} F_{k}(x, y) V_{m 1}(y) \mathrm{d} \nu_{\Delta}(y)\right)^{p} U_{l}(x) \mathrm{d} \mu_{\Delta}(x)\right]^{\frac{s}{p}}}{\left[\int_{X}\left(\int_{Y} \mathcal{G}_{t}(x, y) V_{m 1}(y) \mathrm{d} \nu_{\Delta}(y)\right)^{q} \mathcal{W}_{n}(x) \mathrm{d} \lambda_{\Delta}(x)\right]^{\frac{s-1}{q}}} \\
& +\frac{\left[\int_{X}\left(\int_{Y} F_{k}(x, y) V_{m 2}(y) \mathrm{d} \nu_{\Delta}(y)\right)^{p} U_{l}(x) \mathrm{d} \mu_{\Delta}(x)\right]^{\frac{s}{p}}}{\left[\int_{X}\left(\int_{Y} \mathcal{G}_{t}(x, y) V_{m 2}(y) \mathrm{d} \nu_{\Delta}(y)\right)^{q} \mathcal{W}_{n}(x) \mathrm{d} \lambda_{\Delta}(x)\right]^{\frac{s-1}{q}}} \\
& -\frac{\left[\int_{X}\left(\int_{Y} F_{k}(x, y) V_{m 1}(y) \mathrm{d} \nu_{\Delta}(y)+\int_{Y} F_{k}(x, y) V_{m 2}(y) \mathrm{d} \nu_{\Delta}(y)\right)^{p} U_{l}(x) \mathrm{d} \mu_{\Delta}(x)\right]^{\frac{s}{p}}}{\left[\int_{X}\left(\int_{Y} \mathcal{G}_{t}(x, y) V_{m 1}(y) \mathrm{d} \nu_{\Delta}(y)+\int_{Y} \mathcal{G}_{t}(x, y) V_{m 2}(y) \mathrm{d} \nu_{\Delta}(y)\right)^{q} \mathcal{W}_{n}(x) \mathrm{d} \lambda_{\Delta}(x)\right]^{\frac{s-1}{q}}} \\
\geq & 0,
\end{aligned}
$$

where in the last inequality we used (4.4) from Theorem 4.1. Using Theorem 4.1 again, $V_{m 2} \geq V_{m 1}$ implies

$$
\mathrm{BD}\left(V_{m 2}\right)=\mathrm{BD}\left(V_{m 1}+\left(V_{m 2}-V_{m 1}\right)\right) \geq \mathrm{BD}\left(V_{m 1}\right)+\mathrm{BD}\left(V_{m 2}-V_{m 1}\right) \geq \mathrm{BD}\left(V_{m 1}\right) .
$$

The proof of (5.3) is similar. If (4.5) holds, then the reversed inequalities of (5.1), (5.2) and (5.3) can be proved in a similar way.

Let $F_{k}, \mathcal{G}_{t}, U_{l}, V_{m}, \mathcal{W}_{n}$ be fixed functions. We define a functional $\mathrm{BD}_{1}$ by

$$
\begin{aligned}
\mathrm{BD}_{1}(A)=\int_{A} \frac{\left(\int_{X} F_{k}^{p}(x, y) U_{l}(x) \mathrm{d} \mu_{\Delta}(x)\right)^{\frac{s}{p}}}{\left(\int_{X} \mathcal{G}_{t}^{q}(x, y) \mathcal{W}_{n}(x) \mathrm{d} \lambda_{\Delta}(x)\right)^{\frac{s-1}{q}}} V_{m}(y) \mathrm{d} \nu_{\Delta}(y) \\
-\frac{\left[\int_{X}\left(\int_{A} F_{k}(x, y) V_{m}(y) \mathrm{d} \nu_{\Delta}(y)\right)^{p} U_{l}(x) \mathrm{d} \mu_{\Delta}(x)\right]^{\frac{s}{p}}}{\left[\int_{X}\left(\int_{A} \mathcal{G}_{t}(x, y) V_{m}(y) \mathrm{d} \nu_{\Delta}(y)\right)^{q} \mathcal{W}_{n}(x) \mathrm{d} \lambda_{\Delta}(x)\right]^{\frac{s-1}{q}}},
\end{aligned}
$$

where $A \subseteq Y$.

For $\mathrm{BD}_{1}$, the following result holds.

Theorem 5.2. (i) Suppose $A_{1}, A_{2} \subseteq Y$ and $A_{1} \cap A_{2}=\emptyset$. If (4.2) or (4.3) holds, then

$$
\mathrm{BD}_{1}\left(A_{1} \cup A_{2}\right) \geq \mathrm{BD}_{1}\left(A_{1}\right)+\mathrm{BD}_{1}\left(A_{2}\right)
$$

and if (4.5) holds, then the above inequality is reversed.

(ii) Suppose $A_{1}, A_{2} \subseteq Y$ and $A_{1} \subseteq A_{2}$. If (4.2) or (4.3) holds, then

$$
\mathrm{BD}_{1}\left(A_{1}\right) \leq \mathrm{BD}_{1}\left(A_{2}\right)
$$

and if (4.5) holds, then the above inequality is reversed.

The proof of Theorem 5.2 is omitted as it is similar to the proof of Theorem 5.1. 
Remark 5.1. If $S_{k} \subseteq X$ has $k$ elements and if $S_{m} \supseteq S_{m-1} \supseteq \ldots \supseteq S_{2}$, then (4.2) or (4.3) implies

$$
\mathrm{BD}_{1}\left(S_{m}\right) \geq \mathrm{BD}_{1}\left(S_{m-1}\right) \geq \cdots \geq \mathrm{BD}_{1}\left(S_{2}\right) \geq 0
$$

and $\mathrm{BD}_{1}\left(S_{m}\right) \geq \max \left\{\mathrm{BD}_{1}\left(S_{2}\right): S_{2}\right.$ is any subset of $S_{m}$ with 2 elements $\}$, while (4.5) implies the reversed inequalities with max replaced by min.

Conflicts of Interest: The author(s) declare that there are no conflicts of interest regarding the publication of this paper.

\section{REFERENCES}

[1] M. Anwar, R. Bibi, M. Bohner, and J. Pečarić, Integral inequalities on time scales via the theory of isotonic linear functionals, Abstr. Appl. Anal. 2011(2011), Art. ID 483595.

[2] R. Bibi, M. Bohner, J. Pečarić, and S. Varošanec, Minkowski and Beckenbach-Dresher inequalities and functionals on time scales, J. Math. Inequal. Appl. 2013(2013), 299-312.

[3] M. Bohner and A. Peterson, Dynamic equations on time scales: An introduction with applications, Birkhäuser, Boston, 2001.

[4] M. Bohner and G. Sh. Guseinov, Multiple integration on time scales, Dynam. Systems Appl. 14 (2005), 579-606.

[5] M. Bohner and G. Sh. Guseinov, Multiple Lebesgue integration on time scales, Adv. Difference Equ. 2006 (2006), Art. ID 26391.

[6] B. Guljaš, C. E. M. Pearce, and J. Pečarić, Some generalizations of the Beckenbach-Dresher inequality, Houston J. Math. 22 (1996), 629-638.

[7] S. Hilger, Ein Maßkettenkalkül mit Anwendung auf Zentrumsmannigfaltigkeiten, Ph. D. thesis, Universität Würzburg, 1988.

[8] S. Hilger, Analysis on measure chains - a unified approach to continuous and discrete calculus, Results Math. 18 (1990), $18-56$.

[9] B. Ivanković, J. Pečarić, and S. Varošanec, Properties of mappings related to the Minkowski inequality, Mediterranean J. Math. 8 (2011), 543-551.

[10] S. Varošanec, A generalized Beckenbach-Dresher inequality and related results, Banach J. Math. Anal. 4 (2010), 13-20. 